\title{
Über Heliumgehalt und Radioaktivität von Erdgasen.
}

\author{
Von
}

Emerich Czakó.

Mit 2 Figuren im Text.

In Ergänzung der ausfuhrlichen gasanalytischen Untersuchang von europäischen Erdgasen, welche im vergangenen Jabre im hiesigen Chemisch-Technischen Laboratorium auggefuhrt wurde, ${ }^{1}$ bot sich Gelegenheit eine Anzahl von natürlichen kohlenwasserstoffhaltigen Gasausströmungen auf ibr Edelgasgehalt zu prüfen, entsprechende Versuchsmethoden durchzuarbeiten und Heliumbestimmungen auszufahren. Wegen der Eigenartigkeit solcher Experimentaluntersuchungen and weil dieselben als Grundlage und Anregung zur Ausfuhrung weiterer Untersuchnngen geeignet sein durften, sei im folgenden eine genauere Schilderung derselben gegeben. Im Laufe der Arbeit bot sich ferner Gelegenheit, im Rahmen einer Studienreise Messungen uber die Radioaktivität einer Reihe von europäischen Erdgasen auszaführen, wobei durch den engen $\mathrm{Zu}$ sammenhang, welcher zwischen den beiden Erscheinungen besteht, für die Heliumbestimmungen eine über die einfache gasanalytische Bedeutung der Frage hinsusgehende Grondlage geboten wurde and für die gemeinsame Diskussion der erhaltenen Resultate sich erweiterte Gesichtspunkte eröffneten.

Die Untersnchungen schlossen sich unmittelbar an die ron Stereking und Latterschliger an, die im hiesigen Chemischen Institute die Bestimmung des Heliumgehaltes ron badischen Thermalquellengasen unternommen haben, wortber kẗrzlich publiziert wurde. ${ }^{2}$

\section{I. Über Heliumgehalf.}

Die Auffindung des Heliums in natũrlichen Gasausstrōmungen folgte kurz der ersten Isolierung dieses Gases aus dem Mineral Cleveit durch RAMSAY ${ }^{3}$ im Jahre 1895. AuBer einer groBen Reihe verschiedener Mineralien wurden zahlreiche Quellwässer bzw. die in

1 E. Cz^zó, Beitræge zur Kenntnis natïrlicher Gasquвströmungen. Dr.Ing.-Disgertation, Techn. Hochschule Karlsruhe 1913, Braunsche Hofbuchdruckerei u. Verligg.

${ }^{2}$ Sievering und Lautengchlïger, Physikal. Zeitschr. 18 (1912), 1043.

- Ramsat, Chem. Newo 71 (1895), 151.

Z. anorg. Chern. Bd. 82. 
letzteren gelösten Gase auf Heliumgehalt untersucht. Bald unterzog man auch die aus dem Quellwasser spontan entweichenden Quellgase selbst einer Untersuchung [RAyLeigh und RAMSAx, die Gase der Königsquelle in Bath (1896), Borchand und Troost, diejenigen einiger Thermalquellen in den Pyrenäen (1895) und Kayser die Quellgase von Wildbad im württembergischen Schwarzwald (1895)], und den interessanten Ergebnissen dieser ersten Untersuchungen folgte bald eine bis in die Gegenwart bestehende emsige Forschung in derselben Richtung. Es erwies sich, daB der allergröBte Teil dieser Quellgase heliumhaltig ist und auf diese Weise der Atmosphäre ständig, wenn auch nur in geringen Mengen Helium zugeführt wird.

Untersuchungen über den Edelgas- und insbesondere Heliumgehalt von kohlenwasserstoffhaltigen Gasausströmungen lagen bisher nur sehr vereinzelt ror. Zuerst untersuchte M. Schloesing fils ${ }^{1}$ im Jahre 1896 Schlagwetter der Steinkohlengruben von St. Etienne und Plat-de-gier auf Argongehalt; den Heliumgehalt hatte er noch nicht berücksichtigt. In neuerer Zeit setzte Moureu (zusammen mit LEPAPE), gestützt auf Erfahrungen, die er gelegentlich der Untersuchung zahlreicher französischer Thermalquellengase gesammelt hat, ${ }^{2}$ die Untersuchungen Schloesing s über Edelgasgehalt von Grubenwetter und Grubengasen in einer wesentlich vollkommeneren Appa. ratur fort. ${ }^{3}$ Von weiteren Untersuchungen, die sich mit dem Nachweis des Heliums in Gasen mit weit höberem Kohlenwasserstoffgehalt befaBten, sind bisher nur diejenigen von $\mathrm{C}_{A D Y}$ und MAC FARLAND (1907) über zahlreiche nordamerikanische Erdgase, sowie von Voller und WaLter (1910) über den Helium- und Argongehalt des Neuengammer Erdgases bekannt.

Bei diesen Untersuchungen ist die Beseitigung der Koblenwasserstoffe für die experimentelle Ausführung von einiger Wichtigkeit. Bei Grubenluft oder Schlagwetterproben, die nur geringe Mengen, höchstens einige Prozente Methan enthalten, kann die Entfernung desselben durch Verbrennung über glühendem $\mathrm{CuO}$ verhältnismäBig leicht geschehen (Scrlozsing, Mouret). Anders ist es bei den reinen Erdgasen, mit meist über $90 \% \mathrm{CH}_{4}$-Gehalt. Die langsame Ver-

${ }^{1}$ M. Scriorsino fils, Compt. rend. 123 (1896), 233.

2 Eine ausührliche Zusammenfassung seiner zahlreicher Edelgasarbeiten (die seit 1895 in den Compt. rend. erschienen sind) gab Morreu vor kurzem im Journ. chim. phys. 11 (1913), 63-153.

a Modred und Lepape, Compt. rend. 153 (1911), 847. 1043. 
brennung der wegen dem anzuwendenden Anfangsvolumen des Versuchsgases notwendigerweise gröBeren Methanmengen in einer evakuierten Apparatur von möglichst kleinem Eigenvolumen, ist eine ziemlich schwierige und langwierige Aufgabe.

VOLLER und WALTER $^{1}$ verfuhren bei ihrer Ontersuchung des Neuengammer Erdgases in der Weise, daB sie kleinere Gasproben mit überschüssigem elektrolytischen Sauerstoff gemischt zar Explosion brachten, dann die entstandene Kohlensäure in der Kalipipette, den uberschüssigen Sauerstoff durch alkalische Pyrogallollösung absorbierten und diese Manipulation mit neuen Gasproben so oft wiederholten, bis ein genügender, aus Stickstoff und den Edelgasen bestehender Gasrest angesammelt war. Diese wurde in eine Luftpumpe überfuhrt und in einer vorhor evakuierten GerssLERschen Röhre der spektralanalytischen Untersuchung unterworfen. Eine vorhergehende Reinigung des. Edelgasrestes hatten sie nicht vorgenommen; den Stickstoffgehalt hatten sie durch die inneren Magnesiumelektroden der Spektralröhre absorbieren lassen, gestutzt auf Beobachtungen von Troost und OUvRakd, ${ }^{2}$ wonach aus einem Gasgemisch beim Stromdurchgang der Stickstoff durch die Absorptionswirkung des erglühenden Metalles allmählich entfernt wird, während Helium und Argon nur dann absorbiert werden sollen, wenn sämtlicher Stickstoff schon verschwunden ist. Sie konnten durch diese einfache Versuchsausführung den Nachweis erbringen, daB im Neuengammer Erdgas Helium und Argon enthalten ist. Fine quantitative Bestimmung des Edelgasgehaltes haben sie nicht ausgeführt, sondern gestlltzt auf Untersuchungen von CoLise und RAMSAX, ${ }^{3}$ sowie auf eigene vergleichsweise angestellte spektralanalytische Untersuchungen des Edelgasrestes der gewöbnlichen atmosphärischen Luft, nur Schätzungen angestellt. Sie kamen zu dem SchluB, daB der Argongehalt des Gases nur etwa $1 / 20$ von dem der atmosphärischen Luft beträgt, d. b. $\% / 100 \%$, der Heliumgehalt aber $0.01-0.02 \%$ sei, d. h. etwa $100 \mathrm{mal}$ so groB, wio in der Atmosphäre. Wenn auch letzterer Vergleich gegenwärtig nicht mehr zutreffend ist, denn nach neueren Bestimmungen enthält die Luft rund $0.0005 \%$ Helium ${ }^{4}$ und nicht $0.00015 \%$, wie VolLER und W $\triangle$ LTER auf Grund von älteren Bestimmungen angenommen hatten,

1 Voller u. W

2 Troost u. Quorard, Compt. rend. 121 (1895), 394.

8 Collie und Raysay, Proc. Roy. Soc. Lond. 59 (1896), 257.

4 Vgl. S. 261. FuBnote 1-3. 
konnte die Richtigkeit ihrer indirekten, aus dem nur geschätzten Argongehalt des Gases abgeleiteten Angaben über dessen Heliumgehalt durch die weiter zu beschreibende direkte gasvolumetrische Methode bestätigt werden, indem der Heliumgehalt des Neuengammer Erdgases zu $0.014 \%$ gefunden wurde.

Einige Jahre früher schon haben CADY und MAC FARLAND ${ }^{1}$ an der Kansas-Universität in Lawrence zur Untersuchung zablreicher nordamerikanischer Erdgase eine viel exaktere und bequemere $\mathrm{Me}$ thode angewendet; sie beseitigten den Kohlenwasserstoffgehalt der Gasproben durch Abkühlung mit flüssiger Luft auf $-185^{\circ}$ und dadurch bewirkte Kondensation sämtlicher höher siedender Gasbestandteile. Der die Edelgase enthaltende Gasrest wurde dann nach Dewars Methode ${ }^{2}$ der Wirkung von, mit flüssiger Luft gekühlter Kokosnußkohle ausgesetzt, wobei sämtliche verunreinigende Bestandteile des Gasrestes - in der Hauptsache $\mathrm{O}_{2}, \mathrm{~N}_{2}$ und Argon mit den selteneren Edelgasen - $\left(\mathrm{CO}\right.$ und $\mathrm{H}_{2}$ war in den Gasen im allgemeinen nicht vorhanden und auch in sämtlichen von mir untersuchten Gasproben abwesend gewesen) absorbiert werden und im wesentlichen nur Helium zurückbleibt, welches spektroskopisch untersucht und volumetrisch gemessen wurde.

Ich habe diese Heliumbestimmungsmethode auf „kaltem" Wege ihrer Exaktheit und nicht zuletzt ihrer Bequemlichkeit wegen zur Untersuchung der besonders zusammengesetzten Erdgase viel geeigneter gefunden, als die Verbrennung der Kohlenwasserstoffe über glühendem Kupferoxyd. Solche Bestimmungen wurden zu Beginn der Heliumuntersuchungen in jenem Apparat ausgeführt, in welchem Sievering und Lautenschläger im hiesigen Chemischen Institut den Heliumgehalt verschiedener badischer Quellgase bestimmt hatten. ${ }^{3}$ Bei mehreren Versuchen, die mit dem Erdgas von Neuengamme und mit dem von Kissármás ausgeführt worden sind, gelang die Verbrennung des hohen Methangehaltes der Gasproben über Kupferoxyd, wegen der relativ schweren Verbrennlichkeit dieses Gases auch durch zwei hintereinander geschaltete glühende Kupferoxydröhren (Verbrennungsöfen), welche das Gas vor dem Einleiten in die vorher evaḳuierte Apparatur passieren muBte, bei noch so langsamer Überleitung nur unvollständig und der größte unverbrannte Teil des

${ }^{1}$ Cady und Mac Farland, Journ. Amer. Chem. Soe. 29 (1907), 1523.

2 Dewar, Ann. Chim. Phys. [8] 3 (1904), 5.

3 l. c. 
Gases muBte vor dem Einleiten doch durch Alssige Loft knndensiert werden.

Die ron mir benutzte Apparatur zur Bestimmung des Heliumgehaltes von Erdgasen (siehe Fig. 1), wurde im wesentlichen auf Grund der Angaben von $C_{\Delta D Y}$ und MaC FarLand zusammengestellt; besondere Einrichtungen wurden zur Abmessung des angewandten Anfungsrolumens, sowie zur Messung des isolierten Heliums getroffen und die Versuchsausfuhrang genauer durchgearbeitet. Auf Grund der Skizze (Fig. 1) soll der Apparat selbst und die Ausführung eines Versuches im folgenden näher beschrieben werden.

$A$ ist eine mit einem 'Trichteraufsatze versehene 10 Literflasche, in welcher sich die Gasprobe befand; $B$ ist das dazu gehörige NivesugefäB. Das Füllen der Flasche mit dem Versuchsgas geschieht durch den Trichteraufsatz bevor sie noch in die Apparatur eingeschaltet ist. $D$ ist eine Trockenröhre $\mathrm{zu} \% \mathrm{mit} \mathrm{CaCl}_{2}, \mathrm{zu}$ $2 / 3$ mit $\mathrm{P}_{2} \mathrm{O}_{5}$ gefullt. 2 ist ein Dreiweghahn, $E$ ein Quetschhahn zur Regulierung der Gasgeschwindigkeit. $F$ ist ein, beiderseitig mit Hähnen 3 und 4 versehenes, TrAvEr sches Kondensationsgefä $B$, geräumig genug, um in seinem unteren Teile die verflüssigten Kohlenwasserstoffe aufzunehmen. Das spezifische Gowicht des flüssigen Methans ist 0.415 bei $-164^{\circ} \mathrm{C}$; je nach der zu verflüssigenden Gasmenge, sowie nach den Abmessungen des zu verwendenden DEwarschen GefäBes, lassen sich die entsprechenden Abmessungen des Verflüssigers jeweils leicht berechnen. Om Verstopfungen zu vermeiden, ist die Gaseinloitungaröhre $H$ in ihrem unteren Teil erweitert. $J$ ist ein Thermoelement aus Kupfer-Konstantan. $C, D, 2$ und 3 sind durch dickwandige Gummischläuche und Drahtligatur miteinander verbunden, sämtliche ubrigen Teile des Apparates sind in Glas verschmolzen. 4 ist ein doppelt aurchbohrter, 5, 6, 7, 8 und 9 mit Schrägbohrung versehene einfache Hähne, sämtliche mit Quecksilberdichtung. $L$ und $M$ sind die mit je $20 \mathrm{~g}$ KokosnuBkohle beschickte Absorptionsgefube von der Form greBer SpREngelscher Pyknometer. Die Kohle war aus KakosnuBschalen hergestellt, ${ }^{1}$ auf

${ }^{1}$ Die Verkokung der KokosnuBachalen wurde in einem bedeckten Eisentiegel unter Zuhilfenabme eines Mekerbrenners vorgenommen. Wie inzwischen mitgeteilteVersuche von HzuprL und V 4 Trk zeigten, besitzt eine bei niedriger Temperatur, twa bei $600^{\circ}$ bereitete KakosnuBkoble wenentlich gröBere Absorptionsfăhigkeit. Noch wirksamer als diese, fanden sie die mit Blut und Wesser verkittete and ebenfalls bei $600^{\circ}$ verkokte Tierkoble. Vgl. HempgL, Zeitschr. f. Elektrochem. 18 (1912), 724. 
4-6 $\mathrm{mm}$ große Stücke zerkleinert und sorgfältig gesiebt; sie befand sich in den AbsorptionsgefäBen zwischen zwei Glaswollepfropfen.

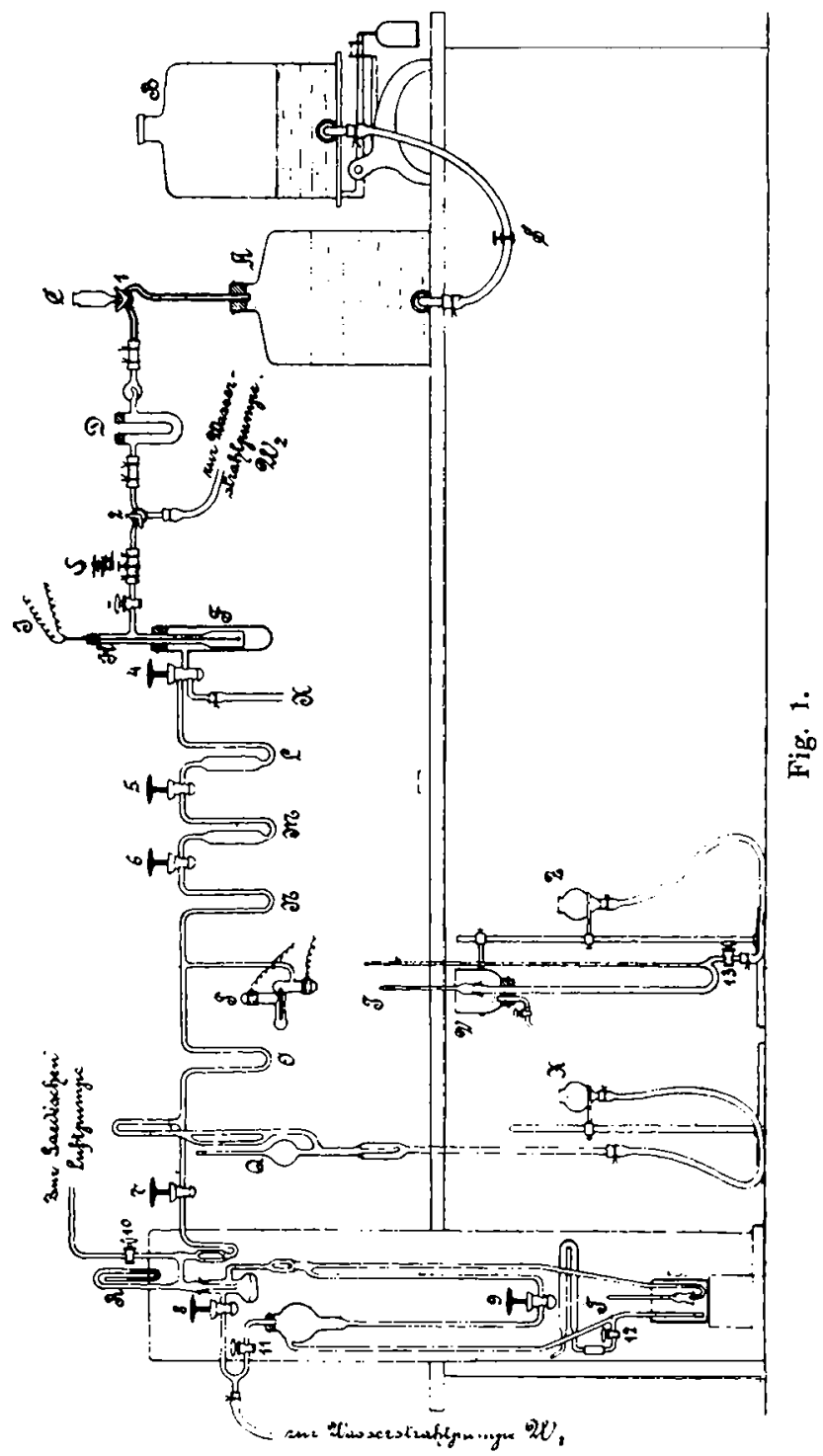

$N$ und $O$ sind ebenfalls durch flüssige Luft gekiühlte U-Röhren, um Quecksilberdämpfe von der Spektralröhre $P$ fern zu halten, was auch auf diese Weise leicht und vollständig gelingt. In Wirklichkeit be- 
fanden sich diese U-Röhren kreuzweise ineinander geschoben hinter der Spektralröhre in demselben DewargefäB. $P$ ist die Spektralröhre mit innerer Kapillare für Länggdurchsicht und elektrolytisch abgeschiedenen äußeren Kupferelektroden (von Goetze in Leipzig). $Q$ ist das Mac Leonsche Manometer mit der Übersetzung von 1:100 und 1:1000. Nach dem Hahn 7 folgte eine für die Zwecke der Versuchsausführung entsprechend abgeänderte, von der Wasserstrahlpumpe $W_{1}$ betriebene automatische SPRENGeLsche Quecksilberluftpumpe ${ }^{1}$ zum Auspumpen des Edelgasrestes, sowie eine elektromotorisch angetriebene GaEDEsche Hochvakuumpumpe zum Evakuieren der Apparatur vor jedem Versuch. Sämtliche unbewegliche Schliffe dieser Luftpumpen wurden mit einem aus 10 Teilen Kolophonium, 1.8 Teilen Olivenöl und 1.1 Teilen venezianischem Terpentin bestehenden Harzkitt gedichtet.

Die Ausführung einer Heliumbestimmung begann mit Ausspülung des gesamten Apparates mit durch Chlorcalcium und Phosphorpentoxyd getrockneter Luft, bei entsprechender Stellung des Dreiweghahnes 2 durch die Wasserstrahlpumpe $W_{2}$ bewirkt. Hierauf wurde unter allmählichem Erwärmen der KokosnuBkohlegefäße bis über $300^{\circ} \mathrm{C}^{2}$ die gesamte Apparatur durch die Wasserstrahlpumpen $W_{1}$ und $W_{2}$ beiderseitig bis zu $14-16 \mathrm{~mm}$ Vakuum evakuiert (Manometer $R$. . Die Hähne 3 und 8 wurden nun geschlossen und mit der Gaedepumpe unter fortwährendem Erhitzen der Kohlegefäbe weiter evakuiert, wobei die von der Kokosnußkohle gelegentlich des vorhergehenden Versuches absorbierten Argon- und Stickstoffmengen allmählich abgegeben wurden. Bei fortgeschrittenerem Vakuum wurde dann auch Hahn 4 geschlossen und die übrige Apparatur bis zum Kathodenlichtvakuum evakuiert, bzw. bis das Spektrum von sämtlichen Linien fremder Gase frei war und auch die beiden hartnäckigsten Spektrallinien des Wasserstoffs verschwunden waren. Der Apparat wurde zwischen den Hähnen 4 und 8 in einem besonderen mehr-

1 In der von BoLrwood angegebenen Anordnung, vgl. Chem. Centrbl. $1897 \mathrm{I}, 450$.

2 Höher zu erhitzen ist nicht ratsam, weil die bei einer direkten Verschmelzung mit den übrigen Apparatenteilen notwendigerweise ebenfalls aus Natronglas zu verfertigende KokosnuBkohlengefäbe sonst im Vakuum Deformationen erleiden können. Die Erhitzung der KohlengefäBe erfolgte in passenden zylindrischen Eisenblechbücbsen, die von unten durch starke Bunsenbrenner erhitzt wurden, oben durch Asbestscheiben bedeckt waren. Die Temperatur wurde durch, bis zur halben Höhe der Blechzylinder eingesteckte Thermometer gemessen. 
tägigen Versuch auf Dichtigkeit geprüt und dieselbe recht befriedigend gefunden. War das höchst erreichbare Vakuum erreicht, so wurden die Luftbäder unter den KohlegefäBen weggenommen, nachdem letztere vollständig erkaitet waren, Habn 10 abgeschlossen und die Gredepumpe abgestellt. Die GefäBe $F, L, M$, sowie die beiden U-Röhren $N$ und $O$ wurden nun in flüssige Luft getaucht und auch die Hähne 5,6 und 7 verschlossen. Jetzt konnte mit dem Einleiten des Versuchsgases begonnen werden.

Inzwischen wurde die Flasche $A$ mit dem Gas gefullt und an die Trockenröhre $B$ geschlossen; letztere wurde dann mit der Wasserstrablpumpe $W_{2}$ evakuiert. ${ }^{1}$ Das Abmessen des angewandten Gasvolumens geschah in der Weise, $\mathrm{da} B$ man den Wasserspiegel der Flasche $B$ mit demjenigen der Flasche $A$ in möglichste Niveaugleichheit brachte und durch den Trichteraufsatz zur Druckeinstellung Wasser einlaufen lieB, bis nichts mehr nachfloB. Der Quetschhahn $S$ wurde nun geschlossen und die Flasche $B$ auf der Dezimalwage gewogen. Nach Öffnen des Quetschhahnes und entsprechender Stellung der Hähne 1,2 und 3 wurde die erforderliche Menge Gas (meist 8-9 l) durch den Überdruck der Niveauflasche in den Ver. flüssiger geleitet, ${ }^{2}$ dann die Druckeinstellung durch den Trichtereinsatz abermals vorgenommen, der Quetschhahn $S$ geschlossen und die Niveauflasche $B$ zurückgewogen. Die Differenz beider Wägungen ergab die eingeleitete Gasmenge auf $10 \mathrm{ccm}$ genau. Das Regulieren der Geschwindigkeit des Ü̉berströmens wurde durch den Quetschhahn $E$ bewirkt. Waren nun 2-3 l Erdgas in den Traversschen Verflüssiger ubergeströmt, ${ }^{3}$ und ihr Kohlenwasserstoffgehalt dort verflüssigt, so wurde Hahn 3 geschlossen und nach kurzem Warten Hahn 4 etwas geöffnet, wodurch das ubber den flüssigen Kohlenwasserstoffen betindliche unkondensierte Restgas, welches neben den Edelgusen noch den größten Teil des ursprünglichen Saverstoff. und Stickstoffgehaltes des Versuchsgases enthielt, in das ge. kühlte und hochevakuierte KohlegefäB $L$ überströmte. Hshn 4 wurde geschlossen, 3 geöffnet und mit dem Einleiten des Versuchsgases

1 Die darin hierbei noch zurückbleibende geringe Luftmenge wird in dem ersten gekühlten Kohlegefäb fast vollstsadig zurückgehalten.

- Bei der Verbinduag des Flascheninhaltes mit der evakuierten Trocken. röhre wurde die in den kapillaren Leitungen des Trichteraufatres befindliche Wassermenge in der kugelförmigen Erweiterung der Trockenröhre aufgefangen.

s Bei Gasproben mit höherem Sauerstoff- und Stickstoffgehalt entsprechend weniger. 
fortgefahren. Inzwischen wurden im KohlegefäB $L$ die vorunreinigenden Gasbestandteile durch die KokoBnuBkohle absorbiert. Bevor nun die zweite Portion des im Thavassachon Gefaße abermals angesammelten Rostgases nach $L$ übergelsasen wurde, wurde dessen in der Hauptsache aus Helium (event. auch Neon) bestehender Gusinbalt durch kurzes Öfnen des Hahnes 5 nach KohlegefäB $M$ tberströmen gelassen und dort abgeschlossen. Nun wird die zweite Portion des Restgases in das KohlegefäB $L$ eingelassen, dann mit dem Einführen des Vereuch\$gases weitergefahren, bis die gewünschte Menge desselben in den Verflüssiger übergeströmt ist, worauf die Hähne 2 und 3 endgultig abgeschlossen werden. Das Verflussigen von 8-9 I Gas dauert etwa $1 \frac{1}{2}$ Stunden. In der weiteren Folge werden nun die GefüBe $F, L$ und $M$ in der oben geschilderten Weise in sinngemäßer Reihenfolge abwochselnd miteinander verbunden, um auf diese Weise das reine Helium im Gefäb $K$ anzureichern. Etwa 1 Stunde nachdem das Versuchsgas eingeleitet wurde, öffnet man kurz dep Hahn 6 und läBt unter ständiger Beobachtung des Spektrums, das Helium in die Apparatenteile $z$ wischen Hahn 6 und 7 ubertreten. Jetzt wird dio automatiache Sprengelpumpe in Tatigkeit gesetzt und nachdem ubor die aufgebogene untere Öfnuan der Fallröbre din quit Quocksilber vollständig luftfrei gefulke kloine MeBburetto $T$-anfgesetzt ist, der Hahn 7 geöffnet und des reine Helium zur rolumetrischen Kemsung in T zusammengepumpt (zeitweise Druckmeseung mit dem Mac LeoDschen Manometer). Rechts rom Hahn 6 wird inzwischen die auch bisher befolgte Manipulation mit den Hähnøn fortgesetzt. Um auch das in den rerflüssigten Kohlenwasserstoffen heläste Helium zu gewinnen, wird zuletzt unter dem Travezsschen Verflüssiger das Dewargefab vorsichtig gesenkt und die flussigen Kohlenwasserstoffe nur soweit erwärmen gelsssen, bis sie einmal sufsieden, worauf wieder in verHiassigte Luft getaucht wird. Ist nun sämtliches Rest. gas nach $L$ ubbergeführt und hieraus das gereinigte Helium in $M$ wieder angesammelt, so wird, nachdem links som Hahn 6 das Helium inzwischen scharf abgepumpt wurde, Hahn 7 geschlossen und Hahn 6 abermals kurz geöffnet, unter Beabschtung des Spektrums des überströmenden Gases. Die eben geschilderten Operationen: d. b. Abpumpen des zwischen Hahn 6 und 7 befindlichen Heliums, nachdem dasselbe auf Reinheit spektroskopisch gepruft wurde, und Anreicherung des Heliums aus dem rechts vom Hahn 6 noch befindlichen Restgasen in dem Kohlegefäß $M$, werden nun ein drittes Mal event. 
auch viertes Mal ausgeführt, wann bei richtiger Versuchsausführung und sinngemäBer Handbabung der Hähne auch sämtliches Helium abgepumpt sein dürfte. Das zuerst und auch bei dem zweitmaligen Öfnen des Hahnes 6 überströmende Helium war in der Regel voll. kommen rein; je nach dem Heliumgehalt des Gases gab es ein mehr oder weniger brillantes und änßerst reines Spektrum mit sämtlichen charakteristischen Linien dieses Gases. Die Beobachtung der Spektra geschah mittels eines einfachen Bunsen schen Spektroskops, dessen Skala mittels GeissLer sche, reines Helium, Argon und Wasserstoff enthaltende Spektralröhren, sowie mit dem Quecksilberspektrum geeicht war. Öfters wurde zur Identifizierung des erbaltenen Heliumspektrums dasselbe mit dem Spektrum der Normal-Heliumröhre verglichen, auch einige photographische Aufnahmen in einem ZEIssschen Spektrographen aufgenommen (vgl. z. B. die untenstehende Photographie des Spektrums des Neuengammer (Gases ${ }^{1}$ ).

Beim drittmaligen und event. vierten Überleiten des Heliums aus Kohlegefä $B$ erschienen einige kaum sichtbare Linien des

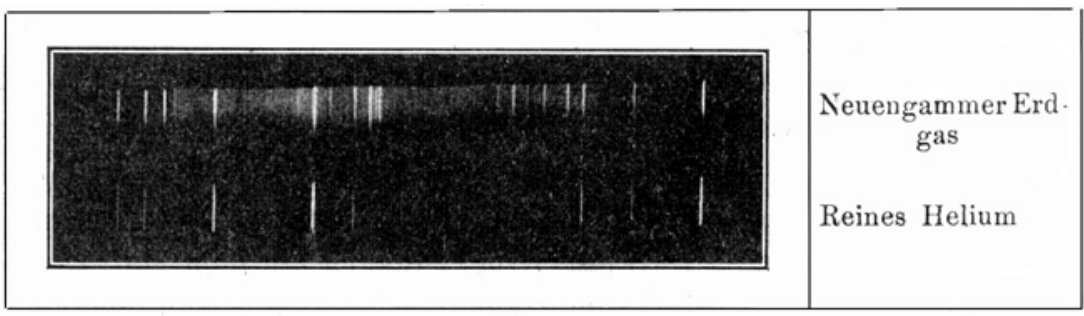

Fig. 2.

Argons; aus den Messungen ron Collie und RAMSAX ${ }^{2}$ ist bekannt, daB in einem Helium-Argongemisch das Spektrum des letzteren bei allen Drucken eben beobachtbar ist, wenn der Argongehalt desselben wenigstens $0.06 \%$ beträgt. Eine demenstprechende Korrektur an dem gemessenen Heliumgehalt anzubringen wäre jedoch nicht angezeigt gewesen, denn bei der dritten und vierten Überleitung des Heliums war die absolute Menge des überströmenden Gases - wie dies aus den mit dem Mac Leopschen Manometer ausgeführten Druckmessungen ersichtlich war - selbst so gering,

1 Die Aufnahmo stammt von einem Versuch, bei welchem die GefuBe $M$ und $O$ nicht gekühlt wurden, um Spektrallinien des Argons und des Quecksilbers zur besseren Identifizierung der Heliumlinie auf die photographische Platte mitzubekommen.

' Collie u. Raysat, Proc. Roy. Soc. Lond. 59 (1896), 257. 
da $B$ eine obigem Argongehalt entsprechende Korrektur weit unter die Fehlergrenzen der Bestimmungen fallen würde. Das im vorgeschritteneren Stadium des Versuches die Linien des Argonspektrums allmählich deutlicher erscheinen, daB also dieses $G a s$ von der gekühlten KokosnuBkohle in geringem Mäbe wieder abgegeben wird, daran ist wohl auch der Umstand schuld, daB die flussige Luft durch die vorzugsweise Absiedung des Stickstoffs ihre Temperatur bekanntlich allmählich ändert; in einem hierfur angestellten Versuch fand ich, daB die Temperatur einer frisch erhaltenen flüssigen Laftprote beim Überfüllen in ein DewargefäB (thermoelektrisch gemessen) $-185^{\circ} \mathrm{C}$ betrag, nach 4 Stunden aber, nachdem dieselbe im offenen GefäB gestanden war, auf $-180^{\circ}$ gesunken ist. In der Regel vergehen aber 3-4 Stunden, vom endgültigen AbschlieBen des Hahnes 3 gerechnet, bis man zum drittmaligen Abpumpen des Heliums kommt und so ist; dă der Siedepunkt des Argons bei $-186^{\circ}$ liegt, das Absorptionsvermögen der Kokosnußkohle aber mit der Temperatur proportional ist, das allmähliche Auftreten von Argonlinien bei der äuBerst intensiven Spektralkraft dieses Gases darch obigen Umstand wohl erklärlich. Fur weitere Versuche empfiehlt es sich daher, das KohlegefäB $M$ durch Eintauchen in unter Vakuum siedende flüssige Luft, auf etwa $-205^{\circ} \mathrm{C}$ abzuküblen. Nicht nur aus diesem einigermaben störenden EinfluB, sondern auch nach Beendigung der Heliumbestimmang und Entfernung der Kublung z. B. unter GefäB $M$, konnte bei sämtlichen Gasproben der Argongehalt auf spektroskopischem Wege direkt festgestellt werden. Von der quantitativen Bestimmung desselben wurde jedoch, teils aus aparativen Gründen, teils weil zu einer Betrachtung des Zusammenhanges zwischen Heliumgehalt und Radioaktivität der Gasproben ihr Argongehalt vor der Hand als belanglos erschien, bei vorliegenden Untersuchungen noch abgesehen.

Das Abpumpen des Heliums von Hahn 6 ab soll, insbesondere nach dem ersten und zweiten Überleiten, möglichst vollständig erfolgen, dann wird nach dem vierten Überleiten und Abpumpen das Helium praktisch genommen bereits vollståndig gewonnen sein.

Die Erscheinung, daB das Intensitätsmaximum des Heliumspektrums bei höberen Drucken im gelben Teil liegt, bei niedrigeren Drucken (etwa $1 / 100 \mathrm{~mm}$ ) aber allmählich in den grünen Teil abergeht, ${ }^{1}$ konnte bei diesem Abpumpen des Gases besonders deutlich

${ }^{1}$ Diese Erscheinung führte Roxar und PAsches (Sitzangsber. d. Akad. d. Wissensch. Berlin 1895, S. 639. 759) zu der sich inzwischen als irrtümlich er- 
beobachtet werden. Wie beim Erscheinen des Heliumspektrums in einem Gasgemisch zuerst immer die brillante gelbe Linie $\left(D_{3}=\right.$ 5876 Å.E.) erscheint, so verschwindet beim Evakuieren einer Spektralröhre immer die grüne Linie (5016 Å.E.) zuletzt. nachdem die gelbe Linie schon unsichtbar geworden ist.

Die volumetrische Messung der abgepumpten Heliummenge geschah in dem MeBapparat VTZ (Fig. 1), in welchen die meistens geringen Heliummengen bei konstantem Volumen durch Messung des Druckes bestimmt wurden. Die etwa $5 \mathrm{ccm}$ fassende MeB. bürette $T$ trug bei $0.25,0.5,1.0,2.0$ und $3 \mathrm{ccm}$ feine eingeritzte Ringmarken; sie paBte mit einem Schliff auf das Ende des kürzeren Manometerschenkels, welches durch den Gummistopfen des Quecksilbergefäßes $V$ geführt, ständig mit Quecksilber überdeckt war. Der längere Manometerschenkel hatte denselben inneren Durchmesser (4 mm) wie der kalibrierte Teil der MeBbürette. Die Niveaukugel $Z$ schloB sich in der Biegung des längeren Schenkels vermittels einer mit Habn 13 versehener Ansatzröhre an. Zur Volummessung wurde die MeBbürette aus dem unteren Quecksilbertrog der SPRENGELschen Pumpe in das Quecksilbergefäß des Meßapparates gehoben und auf den Glasschliff aufgesetzt. Nach Öffnen des Hahnes und durch Senken der Niveaukugel wurde das in der MeBbürette befindliche Helium bis auf die nächste Ringmarke expandieren gelassen, auf dieselbe bei aufsteigendem Quecksilber eingestellt und nach Schließen des Hahnes 13 der Niveauunterschied der beiden Quecksilberkuppen gemessen, bei gleichzeitiger Beobachtung der Temperatur und des Barometerdruckes. Aus den hieraus berechenbaren, auf $0^{\circ}$ und $760 \mathrm{~mm}$ reduzierten Heliumvolumens und aus dem ebenfalls auf $0^{\circ}$ und $760 \mathrm{~mm}$ reduzierten $\nabla$ olumens des angewandten Versuchsgases wurde dann der prozentuale Heliumgehalt des letzteren ermittelt. Die untersuchten Gasproben verschiedener mitteleuropäischer Erdgasquellen sowie auch eines Grubengases aus dem rheinisch-westfälischen Gebiet, befanden sich teils in Stahlflaschen komprimiert (Erdgas Neuengamme und Kissármás), teils in großen, bis 501 fassende Glasballons, da auBer den Edelgasuntersuchungen

wiesenen Auffassung, dab das Helium kein einheitliches Gas sei und daB sein Spektrum zwei besondere Liniensysteme enthält (Helium und Parhelium). DaB die Änderung des Charakters der Entladung durch Druckänderung allein hervorgebracht werden kann, hat zuerst Travers dargetan, Proc. Roy. Soc. Lond. 60 (1897), 449. - Vgl. auch Travens, Experimentelle Untersuchung von Gasen 1905, S. 114. 352 . 
auch für die allgemeinen gasanalytischen Untersuchungen größere Gasproben notwendig waren. Die erhaltenen Resultate der Helium. bestimmungen sind in der Tabelle 1 zusammengestellt:

Tabelle 1.

\begin{tabular}{|c|c|c|c|c|}
\hline & $\begin{array}{c}\text { Angew. } \\
\text { ccm } \\
0^{\circ} \mathrm{C} \\
760 \mathrm{~mm} \mathrm{Hg}\end{array}$ & $\begin{array}{c}\text { Gefunden } \\
\text { Helium ccm } \\
0^{0} \mathrm{C}, \\
760 \mathrm{~mm}\end{array}$ & $\begin{array}{l}\text { Entsprechend } \\
\% \text { Helium }\end{array}$ & $\begin{array}{l}\mathrm{N}_{2} \\
\% \%\end{array}$ \\
\hline $\begin{array}{c}\text { Erdgas von Kissármás } \\
\text { (Ungarn) } \\
\text { Erdgas Pechelbronn, Nr. 220 } \\
\text { Grubengas Zeche Gneisenau } \\
\text { Erdgas Wels (Österreich) } \\
\text { Erdgas Neuengamme bei } \\
\text { Hamburg } \\
\text { Gas einer Tiefbohrung in } \\
\text { ElsaB }\end{array}$ & $\begin{array}{r}8910 \\
8760 \\
5990 \\
6450 \\
7930 \\
2960 \\
7730 \\
1400 \\
630\end{array}$ & $\begin{array}{l}0.094 \\
0.165 \\
0.380 \\
0.432 \\
0.706 \\
0.404 \\
1.628 \\
4.80 \\
2.60\end{array}$ & \begin{tabular}{l|c}
0.0010 & imMittel \\
0.0019 & 0.0014 \\
$\ldots \ldots$ & 0.0063 \\
$\ldots \ldots$ & 0.0067 \\
$\ldots \ldots$ & 0.0089 \\
0.0136 & 0.0141 \\
0.0147 & \\
0.35 & \\
0.41 & 0.38
\end{tabular} & $\begin{array}{l}0.73 \\
6.36\end{array}$ \\
\hline
\end{tabular}

Wie aus diesen Versuchsergebnissen ersichtlich, besitzen die untersuchten Gase einen wechselnden, jedoch in sämtlichen Fällen höheren Heliumgehalt als die gewöhnliche atmosphärische Luft. Der Heliumgehalt der letzteren kann nach neuesten Angaben von ModREU, ${ }^{1}$ RAMSAY ${ }^{2}$ und CLAUDE ${ }^{3}$ im Mittel zu rund $0.0005 \%$ an. genommen werden. Die gefundenen Heliumgehalte der untersuchten Erdgase sind im allgemeinen gering; ein besonders hoher Heliumgehalt, wie CADY und MaC FarLand ${ }^{4}$ bei einigen Erdgasen des Staates Kansas (bis zu $1.84 \%$ Helium) oder Movreu ${ }^{5}$ und seine Mitarbeiter bei einer Reihe von französischen Quellgasen (bis zu $10 \%$ Helium) konnte bisher bei den von uns untersuchten Gasausströmungen nicht beobachtet werden. Die genannten Forscher haben gefunden, daB der Heliumgehalt im allgemeinen mit dem Stickstoffgehalt in Zusammenhang steht; die höchsten Heliumgehalte wurden bei den stickstoffreichsten Gasen gefunden. Diese Beobachtung ist im allgemeinen auch für die von uns untersuchten Gase zutreffend, wenn auch eine direkte Proportionalität nicht zu beobachten ist. Bei dem Gase einer Tiefbohrung in ElsaB mit 46.55\% Stickstoff wurde im Mittel $0.38 \%$ Helium gefunden.

\footnotetext{
${ }^{1}$ Morred, Bull. Soe. Ch́im. de France [4] 9 (1911), Nr. $13(0.00054 \%)$.

${ }^{2}$ Ramsa, Chem. Nercs 105 (1912), 133 . (0.00041\%.)

3 Cladde, Compt. rend. 148 (1909), $1454 . \quad(0.00650 \%$.)

4 1. c.

1. c. ferner Compt. rend. 155 (1912), 197.
} 
Der höchste von uns bisher beobachtete Heliumgehalt wurde in dem Gas der Wildbader Thermalquellen aufgefunden; das Gas bestand aus $2.8 \%$ Kohlensäure und $97.2 \%$ Stickstoff samt Edelgasen. Die Untersuchung ergab $0.71 \%$ Helium neben $1.56 \%$ Argon, also zusammen $2.27 \%$ Edelgase. ${ }^{1}$ Den hohen Edelgasgehalt der Wildbader Quellgase hat zuerst $\mathrm{K}_{\mathrm{AYSER}}{ }^{2}$ beobachtet im Jahre 1895; er konnte aus $430 \mathrm{ccm}$ einer Gasprobe $9 \mathrm{ccm}$, Argon und Helium enthaltenden Edelgasrest isolieren, was einem Prozentgehalt von $2.1 \%$ entspricht. Die Übereinstimmung der beiden Bestimmungen kann somit als recht befriedigend bezeichnet werden.

Aus all diesen eben besprochenen Untersuchungsergebnissen, sowie auch auf Grund der Befunde von zahlreichen Untersuchungen über den Heliumgehalt von Quellgasen darf wohl mit großer Wahrscheinlichkeit angenommen werden, $\mathrm{d} a B$ sämtliche aus der Erdkruste stammenden Gasausströmungen heliumbaltig sind.

Bei dieser nunmehr auf zahlreichen Einzelbeobachtungen fußenden Erkenntnis muB aber der Frage eine, über einen qualitativen Charakter entschieden hinausgehende Bedeutung zugeschrieben werden. Da durch eine Reihe von Experimentaluntersuchungen bereits manche Grundlagen zur quantitativen Verfolgung genügend ausgearbeitet sind, oder aber in Erkenntnis der Mängel auf Grund der bisherigen Erfahrungen nunmehr leicht ausgearbeitet werden können, werden diese unter mehr quantitativen Gesichtspunkten auszuführenden weiteren Untersuchungen zur Lösung mancher, die Chemie, Physik und Geologie unseres Erdkörpers betreffenden Fragen herangezogen werden können.

Es möchten hier nur wenige der aufwerfbaren interessanten Fragen berührt werden. $\mathrm{C}_{A D Y}$ und MAC FARLAND teilten zuerst Beobachtungen mit über die RegelmäBigkeit der Heliumgehalte von Gasen eines geschlossenen gasführenden Gebietes in Zusammenhang mit der allgemeinen geologischen Beschaffenheit desselben. Sie fanden, daß zu demselben geologischen Schichtenzug gehörige Erdgase eine gewisse, meist zwischen kleinen Grenzen schwankende GleichmäBigkeit in ihrem Heliumgehalt, sowie auch in ihrem Gesamtgehalt an gesättigten Kohlenwasserstoffen aufweisen. Sie bekamen

${ }^{1}$ Die mit Herren L. Lattenschläger gemeinsam ausgeführte Untersuchung der beiden letztgenannten Gase wurde wegen dem hohen Stickstoffgehalt in dem von Sievering und Lautenschiäaer gelegentlich ihrer Untersuchungen über Heliumgehalt badischer Quellgase benutzten Apparat ausgeführt. Vgl. Phys. Zeitschr. 13 (1912), 1043.

${ }^{2}$ Chem. News 72 (1895), 89; Chem. Ztg. 1895, 1547. 
für die von ihnen untersuchten Kansas-Gasen Iso-Helium- und IsoParafinlinien, die mit den geologischen Schichtenzügen des betreffenden Gebietes sehr gut übereinstimmten. Sie glaubten aus der UnregelmäBigkeit in dem Heliumgehalt auf eine geologische Störung der Schichten und auch umgekehrt, Schlüsse ziehen zu dürfen. Die Bestätigung der Beobachtungen der amerikanischen Forscher durch ähnliche Feststellungen in anderen Gebieten ist zweifelsohne eine sehr interessante und besonders für die geologische Forschung hoch. wichtige Aufgabe. Unsere europäische geologisch gut charakterisierte, wie auch bohrtechnisch weitgehend aufgeschlossene Erdölgebiete (Galizien, Rumänien) mit ihren Erdölgasen, oder das noch gegen. wärtig im Aufschluß befindliche Siebenbürgische Erdgasrevier mit seinen zahlreichen gasführenden Antiklinalzügen ${ }^{1}$ wären geeignetes Terrain für ähnliche Untersuchungen. - Auch MoUREO ${ }^{2}$ macht darauf aufmerksam, daB die von ihm untersuchten stark heliumhaltigen Quellgase der Cote-d'Or einem und demselben geologischen Schichtenzug angehören und weist auf den nahen Zusammenhang dieser beiden Erscheinungen.

Eine andere in Betracht kommende Frage ist der Edelgas- und insbesondere Heliumgehalt unserer Atmosphäre, auf die wir in diesem Zusammenhang natürlich nicht näher eingehen können und nur auf die ausführliche, mit vielen Literaturangaben versehene Zusammenstellung A. Wegeners ${ }^{3}$ über den heutigen Stand unserer diesbezüglichen Kenntnisse hinweisen möchten. - Da die tägliche Gasproduktion einiger neueren Erdgasausströmungen genau bekannt ist, können wir auf Grund unserer Heliumbestimmungen überschlägige Berechnungen über die ausströmenden Heliummengen anstellen, die mit jenen Erdgasen in die Atmosphäre gelangen. Der mächtigen Erdgasquelle von Kissármás entströmten seit ihrer Entdeckung bis zu ihrer im Jahre 1911 stattgefundenen VersčhlieBung täglich rund $860000 \mathrm{cbm}$ Gas mit einem Heliumgehalt von $0.0014 \%$, das sind $12 \mathrm{cbm}$ Helium pro Tag. Da die Gase mehr als $2^{1} / 2$ Jahre (rund 1000 Tage) ungehindert in die Luft gelassen werden nuBten, weil ihre Bändigung und Fassung mit graßen Schwierigkeiten

1 voN Böckн u. Böнm, Bericht über die Resultate der bisher zur Erforschung der Erdgasvorkommen des siebenbürger Beckens vorgenommenen Untersuchungen. I. Teil. Budapest 1911.

2 Modred u. Lepape, Compt. rend. 155 (1912), 198.

3 Wegener, Phys. Zeitschr. 12 (1911), 170. 214. 
verbunden war, ${ }^{1}$ strömten während dieser Zeit etwa $12000 \mathrm{cbm}$ Helium in die Atmosphäre. Die Erdgasquelle von Neuengamme führt noch mehr Helium mit sich; ihre tägliche Gasproduktion beträgt ca. $500000 \mathrm{~cm}$, das entspricht bei einem Heliumgehalt von $0.014 \%$ täglich $70 \mathrm{cbm}$ Helium. Beide genannten Gasquellen sind gegenwärtig abgeschlossen und warten auf kurz bevorstehende nutzbringende Verwertung, wobei ihr Edelgasgehalt wieder in die Atmosphäre gelungen wird, in welcher Weise auch die Verwertung ibres Kohlenwasserstoffgehaltes stattfinden soll. Zwar weisen viele andere Gasquellen, so besonders einige der von Modrev untersuchten Thermalquellengase der Cote-d'Or einen relativ viel höheren Heliumgehalt auf, die ausgeströmten Heliummengen bleiben jedoch hinter denen der eben genannten mächtigen Erdgasausströmungen weit zurück, indem die Ausströmung der Quellgase selbst meist sehr gering ist. In der vor kurzem erschienenen Mitteilung über sehr stark heliumhaltige Quellgase der Cote-d'Or geben z. B. Mouneu and Lepape ${ }^{2}$ folgende Zahlen an, denen ich die von den beiden Erdgasquellen gelieferten Heliummengen angefügt habe:

Tabelle 2.

\begin{tabular}{|c|c|c|c|}
\hline Gasquelle & $\underset{\%}{\mathrm{He}}$ & $\begin{array}{l}\text { Entwick. Gas } \\
\text { jährlich Liter }\end{array}$ & $\underset{\text { jăhrin Helium Liter }}{\text { Darin }}$ \\
\hline Santenay, Source Lithium & 10.16 & 51000 & 5182 \\
\hline " $"$ Carnot & 9.97 & 179000 & 17845 \\
\hline " Fontaine Salée & 8.40 & $\overline{18250}$ & $\overline{1080}$ \\
\hline & $\begin{array}{l}0.92 \\
1.84\end{array}$ & $\begin{array}{r}18250 \\
547500\end{array}$ & $\begin{array}{r}1080 \\
10074\end{array}$ \\
\hline Nèris, Source César & 0.97 & 3504000 & 39990 \\
\hline La Bourboule, Source Chourey & 0.01 & 30484800 & 3048 \\
\hline & & täglich, cbm & \\
\hline $\begin{array}{c}\text { Erdgas Kissármás Bohrloch II } \\
\text { Erdgas Neuengamme }\end{array}$ & $\begin{array}{l}0.0014 \\
0.0141\end{array}$ & $\begin{array}{c}860000 \\
500000\end{array}$ & $\begin{array}{r}4350000 \\
25550000\end{array}$ \\
\hline
\end{tabular}

E. ErdmanN ${ }^{3}$ berechnete die Heliummengen, welche mit dem wasserstoff haltigen Gas des Kalisalzbergwerkes in Leopoldshall in $4^{1} 1 / 2$ Jahren ausströmten, zu etwa $12 \mathrm{cbm}$; die Gas\&usströmung war dort 31 pro Minute und der Edelgasgehalt (in der Hauptsache Helium, daneben etwas Neon) betrug ca. $0.17 \%$.

1 Vgl. Journ. f. Gasbeleuchtg. 1911, 1251. Die Naturgase Siebenbürgens und die Erdgasquelle von Kissármás.

${ }^{2}$ Moureu und Lepape, 1 . c.

3 E. Erdmanw, Kali 4 (1910), 139. 
Eine weitaus wichtigere Heliumquelle für die Atmosphäre dürften aber wegen den überaus großen ausströmenden Gasmengen die Grubenwetter bilden; es soll nur auf den Umstand hingewiesen werden, daB z. B. allein im Ruhrbezirk, und nur aus den 161 Anlagen, auf welchen bestimmbare Schlagwettermengen ausströmten, täglich ca. 600 Millionen cbm Wetterluft aus den Gruben ventiliert wurde mit einem durchschnittlichen Methangehalt von $0.2 \%$, was einer Methanmenge von täglich $1200000 \mathrm{cbm}$ entspricht, ${ }^{1}$ die gelegentlich des Abbaues der Kohlenflötze frei geworden sind. Der Edelgas- und insbesondere der Heliumgehalt solcher Gasausströmungen wurde bisher nur wenig untersucht. Erwähnt wurden schon die früheren Untersuchungen von Schloksing, ${ }^{2}$ die in neuerer Zeit von Modred und Lepape fortgesetzt wurden. Das Hauptergebnis dieser Untersuchungen ist der beobachtbare beträchtliche Heliumgehalt der Grubengase (bis zu $0.05 \% \mathrm{He}$ ); neben dem Helium konnten sie auch die Gegenwart der übrigen Edelgase feststellen. Eine andere hiermit zusammenhängende Frage ist die Herkunft der Edelgase, die in den Grubengasen beobachtet worden sind. Über die Herkunft des Argons in den von ihm untersuchten zahlreichen Grubengasen hat schon Schloesing Betrachtungen angestellt; da er nachweisen konnte, daß das Verhältnis zwischen Argon und Stickstoffgehalt der Gasproben und der der gewöhnlichen atmosphärischen Luft fast genau dieselbe ist $(1.18 \%$ statt $1.19 \%)$ schloB er hieraus auf eine den heutigen völlig ähnlichen Zusammensetzung jener ,fossiler" Luft, aus welcher bei der Steinkohlenbildung die Atmosphäre bestand und deren Stickstoff und Argonreste (,fossiles Argon") in den Schlagwettern bzw. in den von der Steinkohle eingeschlossenen Gasen nachweisbar sind.

Was den Ursprung des Heliums in den Grubengasen und auch in den übrigen Gasausströmungen betrifft, so kommen wir gleichzeitig zu der Frage der Radioaktivität derselben, mit der ihr Heliumgehalt in engstem genetischen Zusammenhange steht.

\section{Radioaktivität.}

Indem Beobachtungen über Radioaktivität von kohlenwasserstoffhaltigen Gasausströmungen bisher nur vereinzelt vorlagen,

1 Diese Angaben stammen aus dem Jahre 1898. Vgl. das Werk: „Die Entwickelung des Niederrheinisch-westfälischen Steinkohlenbergbanes in der zweiten Hälfte des 19. Jahrhunderts", Bd. 6, S. 84 u. 86.

${ }^{2}$ Vgl. S. 250.

Z. anorg. Cbem. Bd. 82 . 
wurden anschließend an die Untersuchungen über Heliumgehalt Messungen über Radioaktivität einiger europäischer Erdgasquellen im Laufe des Sommers 1912 unternommen.

Von den verschiedentlichen Gasausströmungen der Erdkruste wurden auf Emanationsgehalt bisher besonders die mit den Wasserquellen zusammen vorkommenden Quellgase vielfach untersucht. Von den zahlreichen Untersuchungen haben für vorliegende Arbeit besonders diejenigen ein Interesse, welche neben der Radioaktivität der Gase gleichzeitig mit dem Heliumgehalt derselben sich beschäftigten. Eine besondere Beachtung glauben wir den ausgedehnten Untersuchungen von Moored über zahlreiche französische Thermalgase beimessen zu dürfen. Er bestimmte mit seinen Mitarbeitern Bicquard und Lepape den Edelgas- und besonders den Heliumgehalt dieser Gase und untersuchte dieselben in Gemeinschaft mit Corie u. Laborde und Brochet auch auf ihren Emanationsgehalt. Es erwiesen sich fast sämtliche Quellgase als helium- bzw. emanationshaltig. Eine direkte Proportionalität zwischen diesen beiden Erscheinungen konnte nicht festgestellt werden. ${ }^{1}$ Auf seine SchluBfolgerungen und Betrachtungen über den Ursprung des Heliums in den Gasausströmungen müssen wir nach der Besprechung der eigenen MeBresultate noch zurückkommen.

Gasausströmungen, welche nicht mit Wasserquellen in Zusammenhang stehen, wurden bisher auf Radioaktivität nur vereinzelt untersucht. Es können nur einige Untersuchungen von Erdölgasen (BøRToN, Hrmstedt, Hurmuzescu) von nordamerikanischen Naturgasen(MAC LenNAN), sowie von Gasausströmungen, welche mit Steinkohlenlagern in Zusammenhang stehen (v. D. BoRne, Movrev) erwähnt werden. Da diese Gase alle kohlenwasserstoffhaltige Gasausströmungen sind, wollen wir die Besprechung dieser Untersuchungen gleichzeitig mit der, der zunächst zu beschreibenden eigenen Messungen vornehmen.

Die Messungen wurden mit einem Engler-SiEvkKingschen Fontaktoskop ausgeführt. Mit Ausnahme des Neuengammer Erdgases wurden die Messungen immer an der Quelle selbst vorgenommen. Die Ausführungen der Messungen geschah in der von Engrer u. Sievering angegebenen Weise. Nachdem der Normalverlust des Apparates bestimmt wurde, wurde die Blechkanne mit

1 Vgl. die ausführlichen Veröffentlichungen von Mourev, Bull. Soc. Chim. de France [4] 9 (1911), Nr. 13, I-XXIV; ferner Moured, Journ. chim. phys. 11 (1913), 63-153. 
dem zu untersuchenden Gase verläßlich ausgepült und gefüllt. Dies konnte bei den meisten Quellen sehr leicht und in kurzer Zeit bewirkt werden, da die Gase unter - oft sogar bedeutendem - Druck frei in die Atmosphäre strömten. Aus diesem Grunde wurde auch die Bestimmung des Normalverlustes, sowie die Ausführung der Bestimmung selbst in einiger Entfernung von den Gasquellen vorgenommen. Die Feststellung der Art des Emanationsgehaltes durch direkte Messungen über Abklingung der Zerfallsprodukte konnte in Ermangelung der hierzu notwendigen Ruhe und Zeit im Rahmen der, mit manchen Unbequemlichkeiten rerbundenen Studienreise nicht vorgenQ̨mmen werden. Aus dem Umstande jedoch, daB die Blechkanne nach den Bestimmungen mit strömender Luft durchgespült schon nach $3 / 4-1$ Stunde denselben Normalverlust aufwies wie bevor, möchte es als sehr wahrscheinlich bezeichnet werden, dab die Aktivität der Gase in der Hauptsache von Radiumemanation herrührte. ${ }^{1}$

Die gefundene Aktivität der von mir besuchten Erdgasquellen ist in der umstehenden Tabelle 3 zusammengestellt; sie wurde ausgedrückt durch den Voltabfall pro Stunde in der 10-Liter-Kanne des Engler-Sievering schen Fontaktoskops beobachtet. Die Kapazität des benutzten Elektroskopes betrug an der Normalkanne gemessen $12.3 \mathrm{~cm}$. Es wurden stets zwei Ablesungen gemacht und deren Mittel genommen. Eine Korrektur wegen Löslichkeit der Emanation, sowie wegen der induzierten Aktivität wurde nicht angebracht, und deswegen auch die Umrechnung auf absolute Einheiten bzw. Curies unterlassen. Die erhaltenen Werte geben jedoch auch in der gewählten Ausdrucksweise ein im relativen Sinne richtiges und anschauliches Bild über die GröBe der Aktivitäten.

Zur Erläuterung der tabellarischen Zusammenstellung muB folgendes bemerkt werden:

1 Die Halbwertzeit der Thoriumemanation beträgt nur 53 Sekunden gegenüber von 3.85 Tagen der der Radiumemanation, so daB eine eventl. vorhandene Thoriumaktivität, welche bei der groBen Verbreitung des Thoriums in der Erdkruste angenommen werden könnte, den Messungen und Beobachtungen leicht entgehen kann, zumal eine statische Mebmethode, wie auch die ExalerSieveringsche, zur Messung von rasch abklingenden Aktivitäten nicht ohne weiteres geeignet ist. Die Halbwertzeit der aus der Emanation entstehenden aktiven Niederschlage des Thoriums ist aber $10 \frac{1}{2}$ Stunden, während die des Radiums nur 28 Minuten beträgt, welcher Umstand mit obigen Beobachtungen bestens übereinstimmt. 
Tabelle 3.

\begin{tabular}{|c|c|c|c|c|c|c|}
\hline Nr. & $\begin{array}{c}\begin{array}{c}\text { Datum } \\
\text { der } \\
\text { Messung }\end{array} \\
\end{array}$ & Gasquelle & $\begin{array}{c}\text { Tiefe der } \\
\text { Bohrung } \\
\mathrm{m}\end{array}$ & $\begin{array}{l}\text { Gasliefe- } \\
\text { rung täg- } \\
\text { lich, cbm } \\
\end{array}$ & $\begin{array}{l}\text { Volt } \\
\text { stund - } \\
\text { Abfall }\end{array}$ & $\begin{array}{c}\text { He- } \\
\text { Gehalt } \\
\%\end{array}$ \\
\hline 1 & 22.XII. 1911. & Neuengamme bei Hamburg & 247 & 500000 & 580 & 0.0141 \\
\hline 2 & 20. IX. 1912 & Wels (Ober-Österr) Bohrung Scharf & 380 & - & 165 & 0.0089 \\
\hline $\mathbf{3}$ & 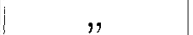 & Krankenhaus & $250-400$ & - & 290 & - \\
\hline 4 & & Herdfabrik & $180-230$ & ca. 1100 & 700 & - \\
\hline 5 & 12. IV. 1912. & Pechelbronn i/Elsaß Bohrung 220 & 275 & - & 300 & 0.0063 \\
\hline 6 & & 1261 & 143 & - & 300 & - \\
\hline 7 & & Gas eines alten Bohrlochs & - & - & 500 & - \\
\hline 8 & 12. IX. 1912. & Kissármás (Ungarn) Bohrloch II & 302 & 860000 & 70 & 0.0014 \\
\hline 9 & $"$ & XII & 225 & 225000 & 50 & - \\
\hline 10 & $"$ & XIII & 108 & 70000 & 120 & - \\
\hline 11 & & $\mathbf{X}$ & 68 & 55000 & 85 & - \\
\hline 12 & 13. IX. 1912. & Bázna (Ungarn) Bohrloch XIV & 140 & 55000 & 125 & - \\
\hline 13 & 14. IX. 1912. & Medgyes (Ungarn) Bohrloch XXII & 102 & 22000 & 140 & - \\
\hline 14 & 16. IX.1912. & Marosugra (Üngarn) Bohrloch V & 1270 & ca. 3600 & 280 & - \\
\hline 15 & 15. IX. 1912. & Campina (Rumänien) Bohrloch 103 & 482 & - & 110 & - \\
\hline 16 & & Gas einer Tiefbohrung in ElsaB & ca. 1000 & - & 17000 & 0.38 \\
\hline
\end{tabular}

Der angegebene Voltstundenabfall des Neuengammer Erdgases wurde durch Multiplikation des im hiesigen Laboratorium gemessenen Wertes mit 2 erhalten, indem das Gas genau 3.85 Tage nach Entnahme der Gasprobe untersucht wurde. Nach freundlicher brieflicher Mitteilung von Herrn Professor B. WALter, Hamburg, wurde eine Messung der Radioaktivität an der Gasquelle selbst bisher nicht ausgeführt.

Im Welser Erdgasgebiet sind zahlreiche Gasquellen von meist nur je einigen $100 \mathrm{cbm}$ täglicher Gasproduktion; die Gase 2-3 solcher verschiedentlicher Bohrlöcher werden in einem gemeinsamen Gasometer aufgesammelt und zur Heizung, Beleuchtung, sowie zur Krafterzeugung in Gasmotoren verwendet. Auffallend ist der ziemlich große Unterschied in der Aktivität der voneinander kaum einige hundert Meter entfernten Gasquellen desselben Gebietes. Äußerst interessant wäre es festzustellen, ob eine entsprechende Änderung auch in den Heliumgehalten der Gase zu beobachten ist, eine Aufgabe, zu deren Lösung ich im Rahmen vorliegender Untersuchungen keine Gelegenheit mehr fand.

Von den untersuchten Pechelbronner Erdölgasen kam dasjenige des Bohrloches Nr. 1261 in Gemeinschaft mit Erdöl vor. Bohrloch Nr. 220 war vor etwa 25 Jahren eine ergiebige Erdölund Gasquelle. Gegenwärtig ist sie versiegt und liefert nur wenig Gase ohne Erdöl. In derselben Größenordnung wurden aktiv gefunden die Gase eines alten verschütteten, zwischen zwei Ölzisternen 
neben der Raffinerie befindlichen Bohrloches. - DaB die mit Erdöl gemeinsam auftretenden Gase emanationshaltig sind, haben zuerst BURTON, ${ }^{1}$ gelegentlich der Untersuchung einiger nordamerikanischer Erdöle, sowie HrMsTeDT ${ }^{2}$ bei der Untersuchung eines elsässischen Errdöls aus Walburg beobachtet. Auch HuRMczescu ${ }^{3}$ konnte feststellen, daB rumänische Erdöle bzw. ihre Gase in verschiedenem MaBe emanationshaltig sind; vgl. auch Nr. 15 der Tabelle 3 der eigenen Messungen an einem rumänischen Erdölgas.

Die siebenbürgischen Erdgase (Nr. 9-15 der Tabelle 3) boten deswegen ein besonderes Interesse, weil sie ein und derselben, etwa $100 \mathrm{~km}$ langer Antiklinale angehören, die sich während ihrer im Jahre 1912 erfolgten bohrtechnischen AufschlieBung als ungemein gasreich erwies. Wie aus den Daten des engeren Kissármáser Gebietes ersichtlich ist (vgl. Nr. 8-11 der Tabelle 3), besteht ein direkter Zusammenhang zwischen der Tiefe der Bohrung und Ergiebigkeit der Gasquelle. Die Aktivität der Kissármáser Erdgase wurde ziemlich gering gefunden, eine allmählich steigende Aktivität konnte jedoch bei den übrigen, in abnehmender Reihenfolge ihrer Ergiebigkeit mitgeteilten Gasausströmungen derselben Antiklinale beobachtet werden. Sollte diese Erscheinung in der Tat eine RegelmäBigkeit darstellen, so könnte es etwa so erklärt werden, da $B$ bei geringer Ergiebigkeit der Quelle, d. h. bei gleichzeitiger geringerer Strömungsgeschwindigkeit der Gase die radioaktive Emanation der durchquerten Bodenschichten von denselben in erböhtem Maße aufgenommen und mitgeführt wird. Von diesem Gasgebiet konnte bisher ebenfalls nur ein Erdgas auf Heliumgehalt untersucht werden.

Als Resultat der Messungen kann festgestellt werden, daB sämtliche der von mir untersuchten Erdgase emanationshaltig sind. Die Stärke der Radiaktivität schwankte in verhältnismäBig kleinen Grenzen und muB im allgemeinen als ziemlich gering bezeichnet werden. Diese Feststellung deckt sich auch mit der allgemeinen geologischen Beschaffenheit der durchgemessenen Gasgebiete. Sie war - zufällig - in sämtlichen Fällen sedimentärer Natur: tonige, mergelige, mit Sandstein wechselnde Schichten in Siebenbürgen und Neuengamme, typische Schlierschichten in Wels. Die sedimentären Bildungen sind aber im allgemeinen viel weniger radioaktiv, somit die aus ihnen hervorgehenden Gase auch weniger emanationshaltig

1 Bunton, Physikal. Zeitschr. 5 (1904), 511.

2 Himstedt, Physikal. Zaitschr. 5 (1904), 210.

- Hormczescu, Petroleum 3 (1907), 235. 
als diejenigen, welche aus Eruptivgesteinen stammen. ${ }^{1}$ Da es nun in der Natur der Entstehung der kohlenwasserstoffhaltigen Gasausströmungen (organische Bildungsweise) liegt, daB sie mit Tiefengesteinen im allgemeinen nicht im primären Zusammenhange stehen, werden auch derartig hohe Emanationsgehalte bei kohlenwasserstoffhaltigen Gasausströmungen, wie sie bei manchen Gasen von Thermalquellen vorzufinden sind, wahrscheinlich kaum zu beobachten sein. Die absolute Tiefe der durchbrochenen Schichten ist für sich genommen auch nicht maßgebend; die gelegentlich meines Besuches in der beträchtlichen Tiefe von $1270 \mathrm{~m}$, doch noch immer in tonig mergeligen Schichten sich bewegende Tiefbohrung Nr. V in Marosugra (Siebenbürgen) erwies sich kaum bedeutend aktiver als die demselben geologischen Gebiet angehörigen Gasausströmungen der höheren Schichten. Sobald jedoch das Urgestein erreicht ist, bekundet sich dies auch in der höheren Aktivität der ausströmenden Gase. Ein interessantes Beispiel lieferte hierzu das Gas in einer Tiefbohrung in ElsaB; die Bohrung lag in nächster Nähe von Gasausströmungen, dessen Aktivität rund 300 Voltstundenabfall betrug. Sie durchbrach jedoch bereits das Urgestein des Gebietes; die zu Anfang 1912 gefundene enorm hohe Radioaktivität, sowie auch ihr bedeutender Heliumgehalt ist in der letzten Reihe der Tabelle 3 angeführt.

Die Aktivität einiger nordamerikanischer Erdgase aus West-Ontario hat MAC LenNax ${ }^{2}$ gemessen; er fand, daß dio aus demselben gasführenden Horizont stammenden Gase gleichen Emanationsgehalt hatten; der allgemeinen geologischen Beschaffenheit des Gebietes entsprechend (kalkhaltige Sedimentärschichten der Silurformation) hat er die Aktivität der Gase ziemlich gering gefunden; aus seinen Angaben berechnete v. D. BoRNE ${ }^{3}$ als höchsten Wert 1.6 und als niedrigsten 0.05 Macheeinheiten, was der Größenordnung nach auch den eigenen MeBresultaten entspricht, wenn man die in „Stundenvoltabfall" angegebenen Werte auf Macheeinheiten umrechnet.

Zur Ausführung ron Messungen über den Emanationsgehalt von Grubengasausströmungen bot sich im Rahmen vorliegender

1 Vgl. Elster und Geiter, Physikal. Zeitschr. 5 (1904), 321. - Gockel, Jahrb. d. Radiaakt. u. Elektronik 7 (1911), 487. - Schumr u. Kurz, Physikal. Zeitschr. 7 (1906), 709. - v. D. BoRne, Zeitschr. d. Deutsch. Geol. Ges. 58 (1906), 1.

2 Mac Lennan, Nature 70 (1904), 151.

${ }^{3}$ v. D. BonNe, Jahrb. d. Radioakt. u. Elektronik 2 (1905), 105. 
Arbeit keine Gelegenheit. Diesbezügliche Messungen sind bisher von v. D. BORNE und von MOUREU mitgeteilt worden; ibre Untersuchungen, einerseits in den Steinkohlengruben des Saargebietes bei Neunkirchen, andererseits in den französischen Gruben zu Liévin ergaben, daB die unmittelbar aus der Steinkohle austretenden Gase keinen Emanationsgehalt besitzen. Diese Tatsache ist in geologischer, wie auch in chemischer Hinsicht von einiger Bedeutung. V. D. BoRNE ${ }^{1}$ wies als erster auf den Umstand hin, dab die Steinkohlenflötze und die aus ihnen entweichenden Gase als auf rein organogenem Wege entstandene Gebilde im allgemeinen völlig inaktiv sind. Nur wenn die Gase bei ihrer Wanderung zur Oberfläche Schichten passieren, in denen radioaktive Zerfallsprozesse vor sich gehen, werden sie mit Emanation beladen, wie er dies auch im Falle eines Bläsergases, welches in der Stormschen Maschinenfabrik in Neunkirchen (bei Saarbrücken) zutage tritt, nachweisen konnte. - Die Untersuchungen Modreus, ${ }^{2}$ soweit sie bisher veröffentlicht worden sind, stellten neben das Fehlen einer radioaktiven Emanation die Gegenwart von Edelgasen und insbesondere von nicht geringen Mengen Helium in den von ihm untersuchten Schlagwetterproben und Grubengasen fest, wie dies auch die Untersuchung des Bläsergases der Zeche Gneisenau zeigte. Es entsteht nun die Frage, in welcher Weise die Entstehung und das Vorhandensein dieses Heliums in solchen Gasausströmungen zu deuten ist und damit zusammenhängend auch die weitere schon früher berührte Frage, ob bei gleichzeitigem Auftreten des Helium- und Emanationsgehaltes einer Gasausströmung irgend welcher, auch rechnerisch verfolgbarer $\mathbf{Z n -}$ sammenhang zwischen diesen beiden Erscheinungen auf Grund des gesammelten Tatsachenmaterials nachzuweisen ist und ob RegelmäBigkeiten $z \mathfrak{u}$ erkennen sind, die für die Betrachtung schon seit mehreren Jahren aufgeworfenen Fragen geologischen und chemischen Charakters von einigem Nutzen oder Bedeutung sein können.

\section{III. Über den Zusammenhang zwischen Radioaktivität und Helium- gehalt von Gasausströmungen.}

Die schon an und für sich recht verwickelten und teils noch unaufgeklärten Verbältnisse der radioaktiven Zerfallsprozesse konnten

1 v. D. Borne, Zeitschr. d. Deutsch. geol. Ges. 58 (1906), 20.

${ }^{2}$ Mouned, Compt. rend. 153 (1911), 847. 1043. 
zwar bei ihrer gesonderten experimentellen Behandlung in Forschungslaboratorien allmählich erkannt und festgelegt werden, ihre Verfolgung im großen Laboratorium der Natur bietet jedoch überaus groBe Schwierigkeiten. Nicht nur, daB die einzelnen radioaktiven Zerfallsprozesse nebeneinander verlaufen können und so die Verhältnisse derselben fast unübersichtlich und unbeherrschbar machen, auch ist mit dem Einflub von chemischen und geologischen Vorgängen des Erdinnern zu rechnen, welche hauptsächlichsten Umstände die Bedingungen derartig komplizieren können, daB man wohl bedacht sein muB, von welcher Seite und unter welchen Umständen man den zu behandelnden Erscheinungen näher zu treten den Versuch wagen soll.

Für die folgenden Betrachtungen wollen wir zunächst nur den bestbekannten radioaktiven Zerfallsprozeb ins Auge fassen nämlich den des Radiums. Neben diesem ist in der Natur auch der Zerfallprozeß des Thoriums weit verbreitet. Um jedoch die Verhältnisse wesentlich $z u$ vereinfachen, soll von der näheren Berücksichtigung des letzteren ${ }^{1}$ gegenwärtig abgesehen und der Gedankengang nur mit dem ZerfallprozeB des Radiums ausgeführt werden. Es möge jedoch hervorgehoben werden, daB für die anzustellende SchluB. betrachtung im wesentlichen gleichgültig ist, ob man einen oder auch mehrere nebeneinander verlaufenden radioaktiven Zerfallsprozesse ins Auge fabt.

Wenn man den Gesamtvorgang des radioaktiven Atomzerfalls des Radiums in die bekannten vier Stufen teilt: 1 . festes radioaktives Ausgangselement, 2. gasförmige aktive Emanation, 3. Gruppe der festen aktiven Niederschläge und 4. gasförmiges inertes Endelement, d. h. Helium, so liegen unter diesen Teilvorgängen die Verhältnisse entschieden bei den beiden gasförmigen Stufen am einfachsten, übersichtlichsten und nach dem Stande unserer gegenwärtigen $\mathrm{MeBmethoden} \mathrm{einer} \mathrm{quantitativen} \mathrm{Verfolgung} \mathrm{am} \mathrm{leichtesten}$ zugänglich. Da der Zusammenhang zwischen radioaktivem Ausgangselement und dem inaktiven Endprodukt des Zerfalls, sowie das Verhältnis dieser beiden zu der aktiven gasförmigen Emanation auch zahlenmäBig genau festgelegt ist, würde durch die Verfolgung der beiden gasförmigen Stadien des Zerfalls, somit durch die diesbezügliche Untersuchung der natürlichen Gasausströmungen, derjenige Weg geboten sein, dessen Beschreitung vielleicht am ehesten weitere Bei-

1 Vgl. auch FuBnote 1 auf S. 267. 
träge liefern kann zur Frage der radioaktiven Vorgänge des Erdinnerns.

Auf Grund seiner zahlreichen Beobachtungen versuchte Moureu vergeblich einen Zusammenhang oder Proportionalität zu finden zwischen Heliumgehalt und Radioaktivität der von ihm untersuchten Quellengase. Aus den eigenen Untersuchungen einiger Erdgase kann - soweit aus den bisher nur wenigen Bestimmungen einige Folgerungen zu ziehen erlaubt ist - eine gewisse RegelmäBigkeit in dem Sinne beobachtet werden, daB die aktirste Quelle auch den höchsten, die schwächste Quelle auch den niedrigsten Heliumgehalt aufwies (siehe Tabelle 3). Es kann auch eine gewisse, wenn auch nur annähernde Proportionalität zwischen den Aktivitäten und dem korrespondierenden Heliumgehalte beobachtet werden; denn nimmt man die Aktivität des Erdgases von Kissármás mit dem dazu gehörigen Heliumwert als Grundlage an, so berechnet sich für die Gase Nr. 5, 1 und 16 der Tabelle ein Heliumgehalt von 0.0060, 0.011 bzw. $0.34 \%$ statt den gefundenen Werten von $0.0063,0.014$ bzw. $0.38 \%$. Allein das Erdgas von WeLS bildet eine starke Ausnahme von dieser RegelmäBigkeit, auf welche nur kurz hingewiesen werden sollte, wenn auch ihre Bestätigung von den Ergebnissen weiterer zahlreicher Bestimmungen abhängig gemacht werden muß. Immerhin kann es von vornherein als wahrscheinlich angenommen werden, daB zwischen Heliumgehalt und Aktivität der Gase eines in sich geschlossenen kleineren Gasterritoriums gewisse Gesetzmäßigkeiten nachzuweisen sein werden, wie solche von $\mathrm{CADY}_{\mathrm{AD}}$ und MAC $\mathrm{F}_{\mathrm{AR}}$ LAND für den Heliumgehalt und von MACLENNAN für die Radioaktivität einiger nordamerikanischer Erdgase gesondert beobachtet werden konnten. Für die Möglichkeit eines nachweisbaren, wenn a uch nur äuBerlichen Zusammenhanges zwischen Heliumgehalt und Radioaktivität von reinen (trockenen) Gasausströmungen gegenüber von Quellengasen sprechen noch die weiteren Umstände, daß 1. bei reinen Gasausströmungen die störenden Einflüsse durch Löslichkeitserscheinungen der Emanation und des Heliums in dem Quellwasser fortfallen ${ }^{1}$ und 2. liegen bei einer reinen Gasausströmung viel genauer

1 Zur Beurteilung eines Quellenkomplexes (Gase, Wasser, eventl. auch Quellsedimente) müBten die erhaltenen Heliumgebalte sowie die Radioaktivitäten des Wassers, Gases usw. jeweils summiert werden, um einigermaBen gleichmäBige Grundlagen zur Feststellung einer eventl. nachweisbaren RegelmäBigkeit zwischen Heliumgehalt und Radioaktivität des Quellkomplexes zu erlangen. Derartige gesonderte Bestimmungen und Summierungen der erhaltenen Werte 
definierte Bedingungen zur Versuchsausführung vor, wenn - wie dies auch bei den eigenen Untersuchungen geschah - die Aktivitätsbestimmungen an der strömenden Quelle selbst, die Heliumbestimmungen hingegen nur dann vorgenommen werden, wenn der Emanationsgehalt bereits abgeklungen ist (etwa $3-4$ Wochen nach der Probenahme des Gases).

Haben wir nun eine heliumhaltige und radioaktive Gasaus. strömung vor uns, die ihren Emanationsgehalt während ihres Durchstreichens durch Erdschichten, in welchen ein radioaktiver Zerfallsproze $B$ vor sich geht, aufgenommen hat, so liegt es wohl am nächsten den Heliumgehalt des Gases als das Endprodukt desselben radioaktiven Zerfallsprozesses anzusehen, von welchem sein Emanationsgehalt herrührt. Diese Annahme ist indessen im allgemeinen nicht zutreffend. Berechnet man nämlich die Mengen Radium, die in gewisser Zeit zerfallen sein mußten, um die im Gas befindlichen oft sehr beträchtlichen Heliummengen zu liefern, so gelangt man zu unwahrscheinlich hohen Zahlen und müBte einen derartig hohen Gehalt unserer Erdkruste an Radium annehmen, welcher bisher noch nirgends beobachtet werden konnte und diese „rezente" Bildungsweise des Heliumgehaltes der Gase äußerst unwahrscheinlich macht. Auf diesen Umstand wurde besonders von Modrev hingewiesen und er hat auch einige Berechnungen ausgeführt.

Viel höhere Zahlen als Moureu für die Quellgase erhalten wir aber bei der Betrachtung der Erdgasausströmungen, die zwar relativ geringen Heliumgehalt besitzen, durch die meist sehr groBe Gasproduktion der Quellen aber viel mehr Helium mit sich führen (vgl. Tabelle 2). Nach den neuesten Bestimmungen und Berechnungen von BOoLTwOOD und RUTHERFORD ${ }^{1}$ beträgt das Heliumvolumen, welches von $1 \mathrm{~g}$ elementarem Radium samt seinen ersten Zerfallsprodukten pro Jahr entwickelt wird: $156 \mathrm{cmm}$; für die beiden Erdgasausströmungen von Neuengamme und Kissármás berechnet sich somit diejenige Menge Radium, welche jährlich zerfallen sein müBte, um die in den Gasen vorhandene und mit ihnen pro Jahr

wurden unseres Wissens bisher nicht ausgeführt. Es ist aber wohl einleuchtend, daB ein Versuch zur Feststellung eines Zusammenhanges allein auf Grund der Untersuchung der Quellwässer oder Quellgase nicht zum Ziele fübren kann; schon die verschiedene Temperatur der Quellen, wie auch der gasreiche oder gasarme Charakter derselben birgt eine Anzahl störender Einflüsse in sich, welche bei den reinen Gasausströmungen gänzlich fortfallen.

1 Boltwood und Rutherford, Phil. Mag. [6] 22 (1911), 586. 
entweichenden beträchtlichen Heliummengen von $25730 \mathrm{cbm}$ bzw. $4380 \mathrm{cbm}$ zu liefern, zu 165000 Tonnen bzw. 28000 Tonnen Radium, wenn der Heliumgehalt ausschlieBlich einer rezenten Bildung zugeschrieben wird. Wenn man weiter bedenkt, welche ungeheueren Heliummengen auch durch die zahlreichen übrigen Gasausströmungen in die Atmosphäre gelangen, ${ }^{1}$ muB man die Entstehung derselben auf rezentem Wege als äußerst unwahrscheinlich, ja fast unmöglich bezeichnen. Nirgends konnten in Mineralien und Gesteinen unserer Erdoberfläche - so tief der Bergmann in diese bisher eindringen konnte - auch nur annähernd ausreichende Radiummengen beobachtet werden. Wir müssen somit nach Mooreu annehmen, daß das Helium bereits fertig gebildet in ungeheueren Mengen in den Schichten der äuBersten erstarrten und erkalteten Erdkruste eingeschlossen und gelagert ist und die Gasausströmungen auf ihrem Wege bis zur Erdoberfläche von diesem „fossilen“ Helium, wie wir dasselbe nach Schloesing und Moured nennen wollen, kleinere oder gröBere Mengen in sich aufgenommen haben.

Zur Annahme eines fertig gebildeten fossilen Heliums sind wir dann auch auf Grund der bereits erwähnten Beobachtungen von v. D. BonNe und Moureu genötigt, nach welchen die unmittelbar aus Steinkohlenflötzen entweichenden Gase keine radioaktive Emanation enthalten, wogegen sie in allen bisher untersuchten Fällen sich als heliumhaltig erwiesen haben. Das Fehlen der Emanation in den auf organogenem Wege entstandenen Humusgesteinen wäre wohl erklärlich; ihr gegenwärtiger Heliumgehalt muB aber dann als fertig gebildet angesehen werden.

Andererseits deutet aber der vorhandene Emanationsgehalt vieler anderer Gasausströmungen auf die noch gegenwärtig verlaufenden Zerfallsprozesse in der Erdkruste, deren gasförmiges inaktives Endprodukt aber meist nur einen verschwindend kleinen Betrag zum Gesamtheliumgehalt der Gasausströmungen liefert, wie dies ans dem bekannten Zusammenhang zwischen Radiumemanation und daraus entstehenden Heliummenge lejcht za berechnen ist.

Der experimentellen Verfolgung des RAmsar-Soddyschen Phänomens, sowie einer Bestätigung der Dewar-Boltwood-Rutherford-

1 Man vergegenwärtige z. B. die Helinmmengen, welche mit den Gasen eines größeren Erdgasterritoriums ausströmen (Untersuchungen von CaDY und MaC FARLAND); auch die, wenn aueh weniger ergiebige doch uuf der ganzen Erdoberfläche verbreiteten Ausströmungen von Quellgasen uud Grubengasen liefern ungeheure Mengen bei ihrer Summierung. 
schen Messungen und Berechnungen über die aus der Emanation entstehenden Heliummengen, durch Verfolgung dieser beiden Erscheinungen bei Naturvorgängen - und wenn auch die denkbar einfachsten, günstigsten und übersichtlichsten Versuchsbedingungen gewählt werden, wie als solche die Untersuchung der reinen Gasausströmungen bezeichnet werden kann - wird man somit schwerlich jemals näher treten können.

Nach diesen Feststellungen muB es noch versucht werden über den Ursprung jener ungeheuren Heliumvorräte der Erdkruste eine Aufklärung zu gewinnen. Wenn wir daran festhalten, daB sämtliches Helium der Erde seinen Ursprung dem Zerfallsproze $B$ irgend eines radioaktiven Elementes verdankt - und hierzu darf man nach dem heutigen Stand der Lehren der Radioaktivität wohl berechtigt sein so sprechen jene groBen Heliummengen von radioaktiven Zerfallsprozessen von ungeheuerer Mächtigkeit, die längst vollständig verklungen und bis zum Endprodukt verlaufen sind. Ob diese Vorgänge von autochtoner Natur waren, d. h. an derselben Stelle verlaufen sind, wo wir gegenwärtig die entsprechenden Heliumgehalte gelagert finden, oder ob die Heliummengen das Produkt von Zerfallsprodukten tieferer Erdschichten und des Erdinnern darstellen, welches entweder als Emanation oder aber, bei der relativ kurzen Lebensdauer der Emanation, wahrscheinlicher als fertiges Helium in die peripherischen Schichten gewandert und dort abgelagert worden ist, kann schwerlich entschieden werden. Auch mag es dahingestellt bleiben, ob nur die uns bekannten radioaktiven Elemente oder ob auch andere längst verschwundene und vollständig zerfallene an jenen mächtigen Zerfallsprozessen teilgenommen haben. Immerhin muB es betont werden, daB bei dem ungeheueren Reich. tum und Vorrat der Erdkruste an fertig gebildetem fossilen Helium auf unserem Planet radioaktive Zerfallsprozesse von ungeheuerer Mächtigkeit stattgehabt haben muBten und in dem Innern unserer Erde auch noch gegenwärtig vor sich gehen. Die Erscheinung, daß besonders die einen vulkanischen Ursprung besitzenden Formationen der Erdkruste als die eigentlichen Träger von radioaktiver Materie und primäre Stätten des radioaktiven Zerfalls in der Erdkruste erkannt worden sind, spricht ebenfalls für obige Annahme. Sei dem aber so, $\mathrm{da} B$ nicht nur auf Grund theoretischer Überlegungen, sondern auch genötigt durch Folgerungen, wie sie aus experimentellen Befunden ableitbar sind, in dem Innern unserer Erde mächtige, dem uns bekannten radioaktiven Atomzerfall ähnliche Zerfallsprozesse 
notwendigerweise anzunehmen sind, so besitzt dieser Umstand für die Wärmeverhältnisse unseres Planeten die gröBte Bedeutung. Aus dem Umstande, daB die Wärmemenge, welche während des Atomzerfalles des Radiums frei wird auf experimenteller Grundlage für $1 \mathrm{~g}$ desselben auf uber $2 \frac{1}{2}$ Milliarden Gramm Kalorien sich berechnet, kann am ehesten ein Begriff gemacht werden über jene gewaltigen Wärmemengen, die gelegentlich der wärmeliefernden Zerfallsprozesse des Erdinnern auftreten und von der die ungeheueren Heliummengen der Erdkruste Zeugen sind. Es muB weiteren mit der letzteren Erscheinung sich befassenden Experimentaluntersuchungen vorbehalten werden, weitere Beiträge und Aufschlüsse zu dieser auch in kosmischer Hinsicht so wichtigen Frage zu liefern.

\section{Zusammenfassung.}

Auf Grund der diesbezüglichen experimentellen Untersuchung von natürlichen kohlenwasserstoffhaltigen Gasen wird der Zusammenhang zwischen Radioaktivität und Heliumgehalt von natürlichen Gasströmungen erörtert. Sämtliche untersuchten Gasproben wurden als helium- bzw. emanationshaltig gefunden; die Versuchsausführung und die erhaltenen Resultate werden genauer besprochen. Aus den gefundenen Heliummengen muB nach dem Vorgange von Movrev auf einen bedeutenden Heliumgehalt der Erdkruste geschlossen werden, welcher, wenn er seinen Ursprung radioaktiven Zerfallsprozessen verdankt, auf ungeheuer mächtige, für die Erklärung der Wärmeverhältnisse unseres Planeten große Bedeutung besitzende ehemalige Zerfallsprozesse des Erdinnern schließen läßt.

An dieser Stelle möchte ich gerne Gelegenheit nehmen, Herrn Professor H. Bunte, in dessen Privatlaboratorium die gasanalytischen Untersuchungen ausgeführt wurden, für seine wohlwollende und die Arbeiten fördernde Unterstützung, wie auch Herrn Professor C. EvaLeR, für wertvolle Anregungen und für die Überlassung von MeBapparaten aufrichtigst zu danken. Herzlichen Dank schulde ich auch Herrn Professor Sievernava für manche Hinweise und für stetes Entgegenkommen, sowie Herrn Dr. LAUTENschläGER für freundliche Mitarbeit bei zahlreichen Edelgasuntersuchungen.

Karlsruhe, Chemisch-Technisches Laboratorium der Technischen Hochschule, Dexember 1912.

Bei der Redaktion eingegangen am 4. Juni 1913. 